\title{
A.I, Robotics, and the Internet of Things for ICT Accessibility and AT
}

\author{
Mada Center
}

Traditionally, assistive technology is considered a separate form of technology designed for persons with disabilities. This kind of technology focuses mainly on tasks like movement, reading, writing, controlling devices, and hearing. However, the modulization of products on the fourth industrial generation is person-centered, not group-centered. This concept allows us to make products usable by as many people as possible. In fact, considering people with all needs in the design process make it adapted to different users. Nowadays, artificial intelligence Al and the internet of things technologies are opening new perspectives in assistive technology and universal design. Together, these two innovative technologies allow the design of products able to learn users' needs through Artificial Intelligence. Thanks to Al, devices became more and more adaptable to users' needs by learning the behavior and interactions of users with them. Internet of things plays also, a fundamental role in the enhancement of the accessibility of devices for people with disabilities.

Robots are undoubtedly the most common application of artificial intelligence. They are used to resolve various spectrums of disability in different sectors or by individuals, especially for individuals who live alone. Robots can be personalized according to the needs of people with disabilities, and this customization ensures that Al and human beings are well-synchronized. Robots are just an instance of the use of artificial intelligence in assistive technology, which makes human life much easier. In fact, they can perform simple tasks, such as making emergency calls when a medical condition occurs, keeping track of appointments and prescriptions, and alerting people to important dates as well. Furthermore, several advanced types of robots can easily control electronic devices at home.

In addition to robots, there are many other impressive examples of this technology that operates for a disabled individual, thanks to artificial intelligence. We quote here only a handful of the most sophisticated applications of Assistive Technology.

\section{Al-Based Visual Aids}

When considering visual difficulties, there are many other solutions, such as contact lenses and glasses. In order to ensure that the focus of the eye's capacity is restored, 


\section{Nafath}

Issue 17 - May 2021

they help to deal with converging and diverging light rays. Thanks to Al and the internet of things, we can do much better than that today. In fact, a research team from the University of Oxford has created smarter glasses. They have invented an augmented reality-based glass that focuses on a particular part of the sight. This has improved the image's contrast and highlighted its characteristics with the aid of AR.

Microsoft invented a Seeing Al application for individuals with visual disabilities. Utilizing this application, the client can hold up his smartphone to an individual and the mobile phone will describe what the individual looks like, what his hair tone is, how old he is, if he looks happy or sad, and so on. By pointing the telephone towards an object, you can understand what the object is, when it expires, etc. The app can also read texts and recognize structural elements, such as paragraphs, headings, and lists.

\section{Al-Based Hearing Aids:}

Artificial intelligence has revolutionized the industry of hearing assistive technology which provided new research opprotunities to enhance hearing capabilities. Hearing aids based on Al can detect the environment and learn what noises the user wishes to focus on. For example, when he is in a noisy area with friends or family, hearing aids with Al can detect and reduce noise and amplify voices. For places that the user visits frequently, hearing aids can remember his preferred settings and automatically turn them on when he goes to such locations.

The production of captions and subtitles is also very important for the deaf to access video and audio content, especially when they are dealing with live communications or online meetings. This ability to automatically recognize speech did not exist before the age of Al. The use of artificial intelligence (Al) based automated speech recognition systems has created possibilities to produce captions and subtitles. Automatic captions are created using speech recognition technology powered by machine learning. Although the technology's accuracy and effectiveness are still improving, it does not provide yet $100 \%$ accuracy and still requires substantial editing. 